\title{
Bacteriophage-based therapy in cystic fibrosis-associated Pseudomonas aeruginosa infections: rationale and current status
}

This article was published in the following Dove Press journal:

Drug Design, Development and Therapy

16 July 2015

Number of times this article has been viewed

\author{
Sami Hraiech ${ }^{1,2}$ \\ Fabienne Brégeon ${ }^{1,3}$ \\ Jean-Marc Rolain'
}

'Institut Hospitalo-Universitaire Méditerranée Infection, URMITE CNRS IRD INSERM UMR 7278, ${ }^{2}$ Réanimation Médicale - Détresses Respiratoires et Infections Sévères, APHM, CHU Nord, ${ }^{3}$ Service d'Explorations Fonctionnelles Respiratoires, APHM, CHU Nord, Marseille, France
Correspondence: Jean-Marc Rolain IHU Méditerranée Infection, URMITE CNRS IRD INSERM UMR 7278, 27 Bd J Moulin, 13385 Marseille Cedex 05, France

Tel +33486 I36828

Email jean-marc.rolain@univ-amu.fr

\begin{abstract}
Pulmonary infections involving Pseudomonas aeruginosa are among the leading causes of the deterioration of the respiratory status of cystic fibrosis (CF) patients. The emergence of multidrug-resistant strains in such populations, favored by iterative antibiotic cures, has led to the urgent need for new therapies. Among them, bacteriophage-based therapies deserve a focus. One century of empiric use in the ex-USSR countries suggests that bacteriophages may have beneficial effects against a large range of bacterial infections. Interest in bacteriophages has recently renewed in Western countries, and the in vitro data available suggest that bacteriophagebased therapy may be of significant interest for the treatment of pulmonary infections in CF patients. Although the clinical data concerning this specific population are relatively scarce, the beginning of the first large randomized study evaluating bacteriophage-based therapy in burn infections suggests that the time has come to assess the effectiveness of this new therapy in CF P. aeruginosa pneumonia. Consequently, the aim of this review is, after a brief history, to summarize the evidence concerning bacteriophage efficacy against $P$. aeruginos $a$ and, more specifically, the in vitro studies, animal models, and clinical trials targeting CF.
\end{abstract}

Keywords: pneumonia, pulmonary infection, bacterial infection, multidrug resistance

\section{Introduction}

Pseudomonas aeruginosa is one of the first pathogens to infect the airways of cystic fibrosis (CF) patients. In the CF lung, a genetic lack of functioning CF transmembrane regulators in the apical membrane of respiratory epithelial cells results in a change in the airway environment that is favorable to $P$. aeruginosa infection. During the evolution of $\mathrm{CF}$-induced respiratory tract disease, $P$. aeruginosa progressively takes the first place among bacteria recovered from pulmonary samples, and up to $80 \%$ of CF adults suffer from chronic $P$. aeruginosa infection. ${ }^{1}$ Chronic pneumonia resulting from $P$. aeruginosa colonization threatens patients with a deterioration in lung function and shortens their life expectancy, ${ }^{2}$ especially when mucoid phenotypes are involved. ${ }^{3}$ Although infection with $P$. aeruginosa may be cured if treatment is started early, ${ }^{4}$ no antibiotic is able to eradicate an established chronic lung infection. Finally, CF patients are exposed to systemic and inhaled broad-spectrum antibiotic treatments early in their life, ${ }^{5}$ which in turn has led to a dramatic emergence of multidrug-resistant (MDR) bacteria and extremely resistant strains of $P$. aeruginosa. ${ }^{6}$ Thus, the "pipeline" of new antibiotics is running dry, ${ }^{7}$ and physicians are facing the threat of untreatable infections.

New antimicrobial molecules or therapeutic strategies against $P$. aeruginosa infections, especially MDR strains, are under investigation. ${ }^{8,9}$ Bacteriophage-based therapy 
appears as a promising "innovative" treatment and deserves a focus. ${ }^{10}$ Numerous bacteriophages active against $P$. aeruginosa have been identified, ${ }^{11}$ and a variety of these phages are efficient against $P$. aeruginosa strains from CF patients. ${ }^{12}$ While the first multicenter randomized controlled study (NCT02116010) evaluating the efficacy and safety of phages against $P$. aeruginosa wound infections in burn patients will soon begin, several steps remain before bacteriophage-based therapies can be applied to CF patients.

After a brief reminder of the history of bacteriophage use, this review summarizes the current data available and the perspectives regarding bacteriophage-based therapy in the treatment of $P$. aeruginosa pneumonia in $\mathrm{CF}$ patients. In the absence of consistent clinical data in this field, we will enlarge the topic to in vitro and animal studies focusing on CF bacterial flora.

\section{Historical context of bacteriophage therapy: a pendulum effect?}

Despite not being demonstrated at the time, Briton Ernest Hanbury Hankin's observation in India in 1896 of antibacterial activity against Vibrio cholerae from the water of the Jumna and Ganges rivers was probably the first experiment due to bacteriophage activity. Later, at the beginning of the 20 th century, the concept of an independent microorganism able to infect and lyse bacteria - namely, the bacteriophage was first co-described independently by two microbiologists, Felix d'Herelle of France and Frederick William Twort of Great Britain. They both questioned whether the agent responsible for bacterial lysis was a bacterial virus destroying bacteria. However, at the time, bacteriophages remained invisible. Soon after, following successful assays on animals as well as himself, d'Herelle pioneered the use of phage preparations to treat patients suffering from Shigella dysentery in France and cholera epidemics in India. ${ }^{13}$ D'Herelle moved then to Georgia, and his collaboration with the scientist George Eliava probably explains why Georgia became the main center of bacteriophage development with the Tbilisi Institute of Bacteriophages, founded in 1923 by Eliava.

Despite controversy about "the therapeutic value of lytic filtrates", bacteriophages were largely produced in the 1940s. In Paris, d'Herelle founded the Laboratoire du bactériophage and commercialized various cocktails of bacteriophages. In the USA, bacteriophages were industrially produced mainly for the treatment of traumatic or postoperative wound infections. However, at the end of the
$40 \mathrm{~s}$, difficulties in maintaining industrial amounts of phage production, treatment failures, and the emergence of antibiotics marked the end of bacteriophage use in Western countries. In the Soviet Union, however, the extensive use of bacteriophage-based therapy persisted, and most of the literature concerning bacteriophages comes from case reports or studies from former USSR countries and Eastern Europe. These studies reported successful treatment of various infections, including Staphylococcus aureus and $P$. aeruginosa, but publications were confined to a small area and, because they lacked control groups, these studies were too weak to convince the rest of the world about phage efficacy and safety.

In the last two decades, bacteriophages regained the interest of scientists in Western countries who face the emergence of MDR bacteria and a decrease in new antibiotics. Characterization of bacteriophages then occurred.

Bacteriophages are ubiquitous organisms present in high numbers in the water, soil, and animal-associated ecosystems. Most of the bacteriophages described to date belong to Caudovirales, which is itself composed of Myoviridae, Siphoviridae, and Podoviridae. These bacteriophages share an icosahedral capsid containing a double-stranded linear DNA and a caudal part with structures for adhesion to bacteria. Lytic phages lead to bacterial death and are weakly implicated in horizontal gene transfer (responsible for antibiotic-resistance gene transfer). This is contrary to the lysogenic bacteriophages ${ }^{11}$ that can integrate into the bacterial genome as prophages and be responsible for virulence factor transfer. Easy to extract, phage isolation is rapid and cost-effective so that any bacterial infection could, in theory, be controlled by bacteriophage-based therapy (Figures 1 and 2). However, the efficacy of the isolated bacteriophages needs verification prior to use, and results obtained in vitro cannot determine effectiveness in vivo, especially when the lung is concerned.

It is only recently that the first well-designed clinical trials were launched and showed encouraging results, mainly for wound infection in burn patients, ulcers, ${ }^{14,15}$ and chronic otitis. ${ }^{16}$ The interest in bacteriophages is so significant that bacteriophages have been elected one of the seven weapons to fight against antibiotic resistance by the US National Institute of Allergy and Infectious Diseases. ${ }^{17}$ In 2014, the first multicenter randomized controlled trial (France, Belgium, and the Netherlands) in humans obtained funding from the European Commission and the trial will be starting soon in patients with wound infections due to Escherichia coli and 


\section{A Test for phage activity against two target bacterial strains}

Lysis plaques

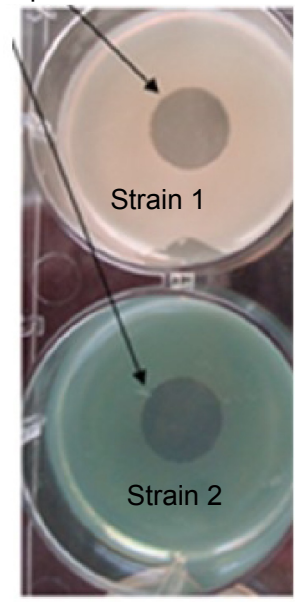

B

Titration of a phage-containing master stock according to double-agar overlay plaque assay

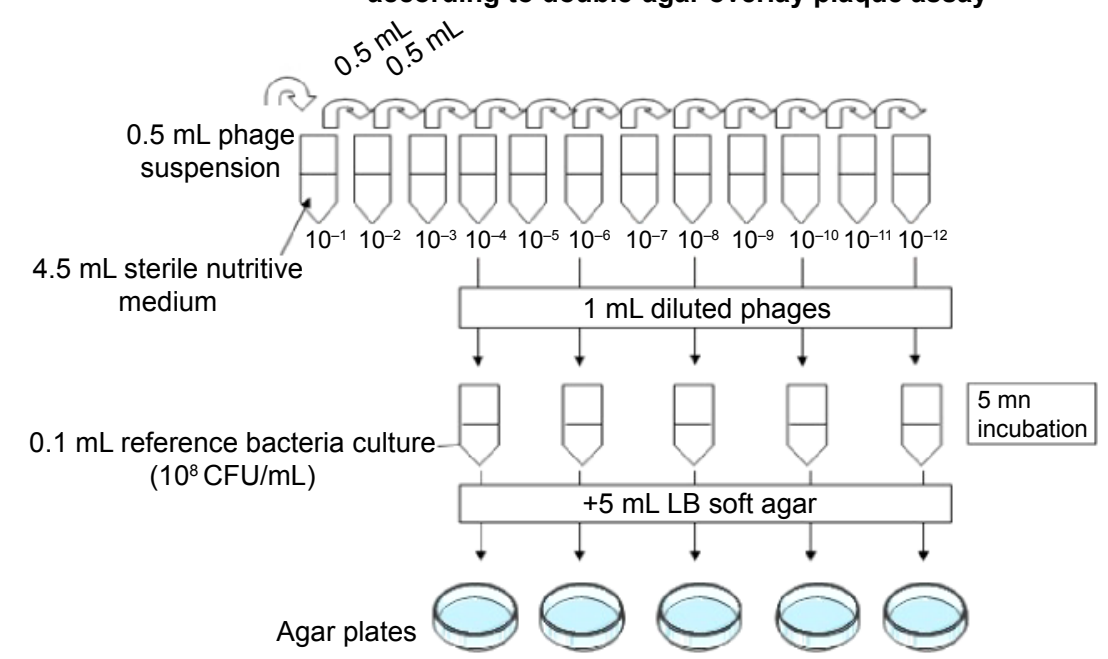

30 minutes drying then $18-24$ hours of incubation at $37^{\circ} \mathrm{C}$

$\rightarrow$ Read the limit dilution with lysis result

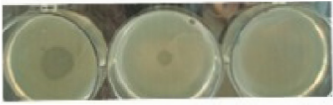

Figure I In vitro steps before phage use for in vitro or in vivo models.

Notes: (A) Specific bacterial activity: the Pseudomonas aeruginosa strains of interest (eg, Strain I and Strain 2) are co-cultured on agar plates with a phage suspension to verify the lytic activity of the phages against known bacterial strains. (B) Phage suspension is titrated with the double agar overlay plaque assay according to Gratia's method ${ }^{68}$ Briefly, serial dilutions of a phage suspension are added to bacteria broth in dilute agar or agarose matrix ("top agar" or "overlay"). After uniform mix, solidification is obtained on standard agar plates ("bottom agar" or "underlay"). After incubation, lysis is visualized as clear areas called plaques and corresponding to decreased density of bacterial lawn due to the lytic activity of the phages. Extrapolation of the initial phage suspension contents is obtained by extrapolation from the last dilution giving a plaque. Phage quantity is therefore expressed as plaque-forming units.

Abbreviations: LB, Luria-Bertani; CFU, colony-forming units.

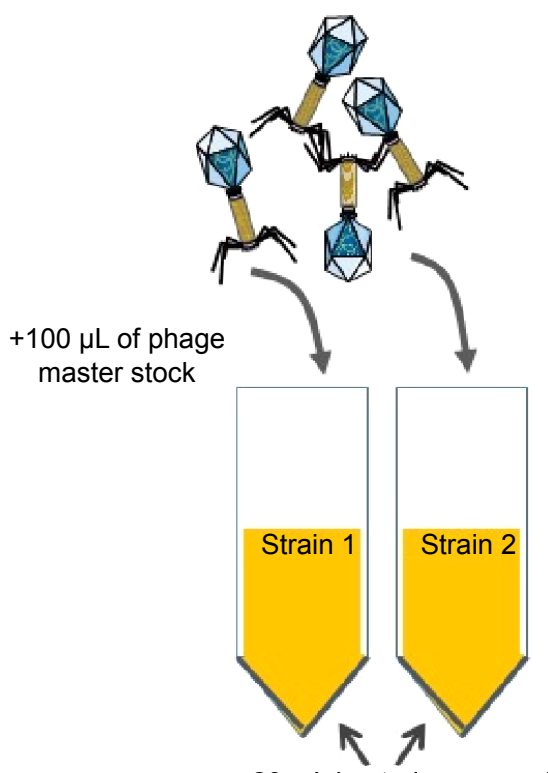

Incubation at $37^{\circ} \mathrm{C}$ for $18-24$ hours centrifugation

$20 \mathrm{~mL}$ bacteria suspension

$\left(10^{8} \mathrm{CFU} / \mathrm{mL}\right)$

Figure 2 Example of Pseudomonas aeruginosa-specific high-titer phage-production protocol. Target strains of $P$. aeruginosa are incubated separately for 24 hours in the early exponential phase $(\mathrm{OD} 600 \mathrm{~nm}=0.1)$ with a known phage cocktail at a 0.035 multiplicity of infection (= volume of phage suspension/volume of bacterial suspension). After 24 hours, the obtained lysates are centrifuged at $5,000 \times \mathrm{g}$ for 30 minutes at $4^{\circ} \mathrm{C}$. Supernatant is filtered through a $0.2 \mu \mathrm{m}$ membrane and stored at $4^{\circ} \mathrm{C}$.

Abbreviations: CFU, colony-forming units; OD, optical density. 
P. aeruginosa to compare phage therapy with silver sulfadiazine (NCT02116010).

\section{Bacteriophage therapy to treat $P$. aeruginosa infections in CF patients: a new paradigm?}

$P$. aeruginosa is a ubiquitous environmental agent. It is also a commensal in humans and is recovered largely in the gut and sometimes the skin. This bacteria acts as an opportunistic agent in CF patients. ${ }^{18}$ One of the major characteristics of $P$. aeruginosa is its ability to develop a biofilm, favoring the development of a niche and conferring a relative protection against host defenses and antibiotics. Moreover, the development of a mucoid phenotype of $P$. aeruginosa is favored. ${ }^{19}$ The biofilm formation associated with $P$. aeruginos a colony development contributes to antibiotic treatment failure, ${ }^{20}$ a feature that was first ascribed to the ability of the biofilm matrix to restrict antimicrobial penetration. ${ }^{21}$ Other mechanisms explaining antibiotic treatment failure include the establishment of slow-growing antibiotic-resistant subpopulations within the biofilm (persister cells) ${ }^{22}$ and biofilmspecific gene expression. ${ }^{23}$ As an alternative to antibiotics, bacteriophages have the advantage of targeting a precise bacterial species. It is known that a specific bacteriophage exists for each specific bacterium. Bacteriophages also produce enzymes active against bacteria. As an example, the endolysin Cpl-1 was delivered by inhalation and was effective to rescue mice with fatal pneumococcal pneumonia. ${ }^{24}$

Given the large amounts of bacteria in CF airways, the CF patient respiratory microbiota is a source of novel phage discovery. It is also plausible that the disease by itself could be a source of new virulent bacteriophages that could be interesting tools to control the CF infectious biomass. Bacteriophages have the ability to penetrate easily into the P. aeruginosa-associated biofilm and are also able to produce natural enzymes that hydrolyze the biofilm, ${ }^{25}$ arguing for their use in chronic $P$. aeruginosa infections in CF patients. As several strains of the same bacterial species often coexist in the same patient, treatment with one bacteriophage at a time may fail, and therefore, cocktails of phages have been developed. For example, the Georgian Pyophage cocktail, synthesized at the Eliava Institute (Tbilisi, Georgia), is a mixture of phages directed against five bacterial species that predominate in purulent infections, including $P$. aeruginosa, and is used for phage therapy. ${ }^{26}$

Prior to their use in humans, experiments using bacteriophage-based therapy were conducted in vitro and in animal experiments, as described following.

\section{In vitro proofs of concept of the efficacy of bacteriophages against $P$. aeruginosa strains from CF patients}

The main in vitro antibacterial effects of bacteriophages against clinical and nonclinical strains of $P$. aeruginosa proven in vivo are summarized in Table 1.

\section{Susceptibility of CF-associated $P$. aeruginosa to bacteriophages}

Observation of bacteriophages from the sputum of $\mathrm{CF}$ patients ${ }^{27}$ has opened the way to extensive research. Following the isolation of a clinical strain of $P$. aeruginosa in a patient sample, the existence of a virulent (lytic) phage targeting this isolate can be assessed from a library of already known phages or can be found de novo. A recent in vitro study assessed the antibacterial efficacy of bacteriophages against CF strains of $P$. aeruginosa ${ }^{28}$ Six different phages were purified from the Georgian Pyophage cocktail, including four genera of bacteriophages with some active against P. aeruginosa. ${ }^{29}$ Phages were tested against 47 strains isolated from CF sputum collected in several French hospitals. Of these 47 strains, 33 were lysed with at least one bacteriophage of the cocktail. The strains that were resistant to the first cocktail prompted an investigation to find new bacteriophages. Two novel viruses were isolated in sewage water from France and the Ivory Coast. The combination of the preexisting different cocktails of phages with the newly identified viruses allowed a larger spectrum against strains of $P$. aeruginosa. This work suggests that it would be possible, from clinical samples from $\mathrm{CF}$ patients, to constitute a new cocktail composition with enlarged antibacterial targets. This is particularly promising considering that clinical strains of $P$. aeruginosa might be more susceptible to bacteriophages than environmental strains. ${ }^{30}$

Very recently, a French team ${ }^{12}$ worked on $P$. aeruginosa strains from CF patients and showed that a specific cocktail of ten phages was able to reduce bacterial growth. The originality of this work was the direct application of the virions on the recovered sputum, suggesting that the phages were active against bacteria in their original environment.

\section{Phage resistance in CF strains of $P$. aeruginosa}

One of the hypotheses to explain phage susceptibility and the virulence of clinical strains issued from CF patients are the clinical characteristics of such strains. Indeed, strains infecting CF in a chronic manner might be more susceptible 


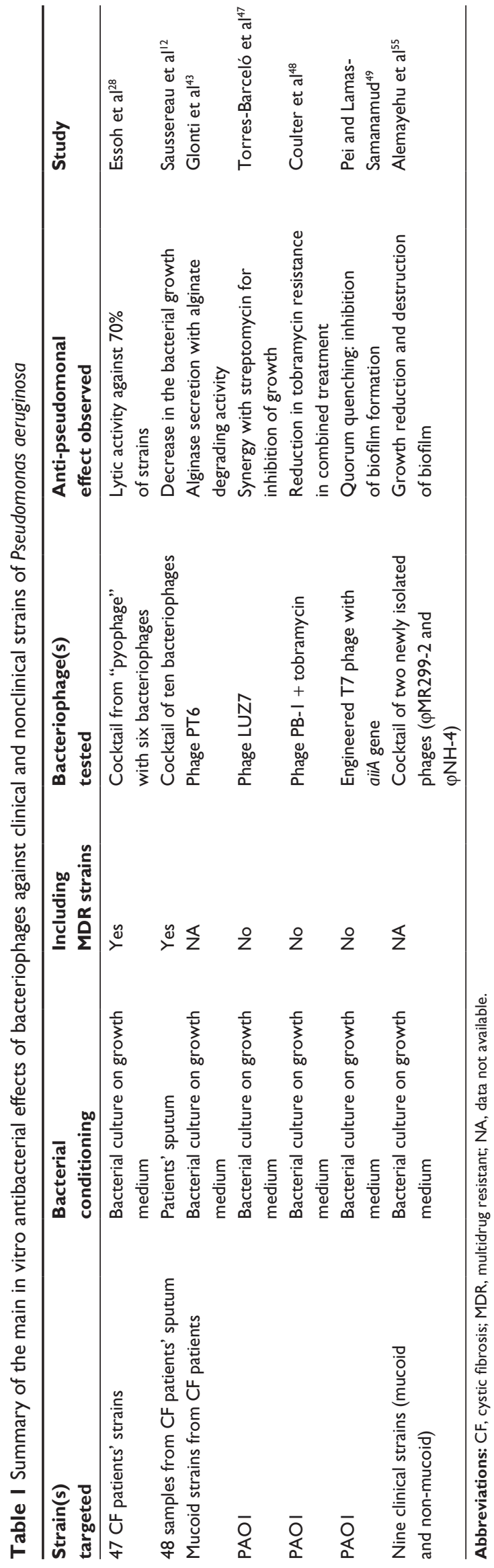

to bacteriophages than strains responsible for intermittent acute infections..$^{31}$ This could be particularly true with strains colonizing the lungs of $\mathrm{CF}$ patients for more than 2 years, probably because their adaptation to the lung environment is associated with a less virulent phenotype with decreased growth and increased susceptibility to bacteriophages. This "fitness cost" has been described with the acquisition of multiple antibiotic resistance. ${ }^{32}$ However, the association between the susceptibility of CF isolates of $P$. aeruginosa toward phages and their clinical characteristics (and, in particular, the duration of lung colonization) was not found in another study. ${ }^{12}$ This particularity remains to be explored.

The mechanisms of bacterial resistance to bacteriophages could involve loss or significant alteration of receptors or overproduction of exopolysaccharides such as alginates. ${ }^{33}$ Bacteriophages have been reported to be largely present in CF lungs. Their identification from lung samples was assessed, but little is known about their densities or biological activity in situ. Chronic CF lungs could be dominated by lysogenic prophages that reside mostly within bacterial genomes but can occasionally convert to the lytic phenotype. ${ }^{34}$ In their lysogenic form, phages might have a relatively small role in regulating bacterial densities despite their prevalence. Moreover, temperate bacteriophages have been implicated in horizontal gene transfer. They have been shown to confer selective beneficial traits to a range of $P$. aeruginosa hosts. ${ }^{29}$ For example, phage $\phi C T X$ infection of the PAS10 strain results in conversion of the bacteria to a toxigenic strain. ${ }^{35}$ The genome sequencing of the $P$. aeruginosa hypervirulent strain LESB58 revealed that it shares 95\% similarity with the laboratory strain PAO1. However, the LESB58 core genome is punctuated by five prophage clusters probably implicated in its highly pathogenic potential. ${ }^{36}$ LESB58 mutants, with disrupted prophage genes, exhibited ten- to 1,000 -fold decreased competitiveness in a rat model of chronic lung infection compared to wild-type LESB58. The choice of lytic phages seems therefore crucial for their use in CF pulmonary infections.

\section{Biofilm inhibition}

One of the main advantages of bacteriophage-based therapy against $P$. aeruginosa is its supposed efficacy against the biofilm. ${ }^{37,38}$ Bacterial biofilms are populations of cells adherent to an abiotic or biotic surface that can grow to be several millimeters thick. ${ }^{39}$ Biofilms formed by $P$. aeruginosa have been found not only on medical implants such as catheters ${ }^{40}$ but also on native airways from CF patients. ${ }^{41}$ Biofilms have been shown to be highly tolerant to antimicrobials, a feature 
that was first ascribed to the ability of the biofilm matrix to restrict antimicrobial penetration. ${ }^{21}$ Other mechanisms that have been described include the establishment of slowgrowing, antibiotic-resistant subpopulations within biofilms (persister cells) ${ }^{22}$ and biofilm-specific gene expression. ${ }^{23}$ In CF patients, the development of a biofilm confers to bacteria a resistance to antibiotics as well as an inhibition of polynuclear chemotaxis.

There are several advantages of bacteriophages over antibiotics in treating biofilm-associated infections. First, in contrast to antibiotics passing over the surface of a biofilm, bacteriophages infect bacteria at the surface and will then replicate, generating a high concentration of antibacterial agents at the site of the infection. Bacteriophages can also infect persister cells and can become active and destroy them when reactivation occurs. Moreover, bacteriophages can produce enzymes that dissolve the biofilm matrix or induce enzyme production by the bacterial host. ${ }^{42}$ This has been shown in vitro using clinical mucoid strains of $P$. aeruginosa from CF sputum. Hanlon ${ }^{25}$ and Glonti et $\mathrm{al}^{43}$ showed that a bacteriophage-derived enzyme was able to depolymerize the alginic acid capsule of $P$. aeruginosa. The alginase produced by these bacteriophages may have the potential to increase the well-being of CF patients by facilitating the expectoration of sputum, accelerating phagocytic uptake of bacteria, and perturbing bacterial growth in biofilms. The ability of bacteriophages to weaken and even destroy the biofilm matrix can also aid the penetration of other agents, raising the possibility of a synergistic combination with antibiotics. Because $P$. aeruginosa is important for producing the biofilm matrix, such synergy could also extend to other species present within a polymicrobial biofilm. While those species might not be targeted by the bacteriophage, they could become more susceptible to antibiotics.

\section{Synergy with antibiotics}

One major concern with $P$. aeruginosa-bacteriophage activity against biofilms is that the bacteriophages have essentially been shown to decrease the biomass of a biofilm but not to eradicate it. ${ }^{44}$ As a consequence, several works have investigated the role of bacteriophages in combination with antibiotics to restore antibiotic activity. ${ }^{45,46}$ Recently, TorresBarceló et al demonstrated the synergy of bacteriophages and streptomycin in the inhibition of the in vitro growth of $P$. aeruginosa. ${ }^{47}$ They showed that the efficacy of bacteriophages associated with streptomycin was superior to the addition of each treatment separately. Moreover, the combination was able to decrease the bacterial growth rebound observed at the end of a single treatment with either bacteriophages or streptomycin. The best synergy was obtained when streptomycin was added 12 hours after the bacteriophage treatment. The resistance to streptomycin and to bacteriophages was also lower in the combined therapy. These results concerning the decrease in antibiotic resistance in combined phage-antibiotic therapies were also recently confirmed using tobramycin and bacteriophages, with a $60 \%$ reduction in tobramycin resistance. ${ }^{48}$

\section{Quorum-sensing inhibition}

Quorum-sensing inhibition is one of the therapeutic options against $P$. aeruginosa infections. Hydrolyzing acylhomoserine-lactones, lactonases have been shown to decrease in vitro biofilm production and in vivo virulence of P. aeruginosa. ${ }^{9}$ A recent study showed that bacteriophages can be genetically modified to produce a lactonase implicated in $P$. aeruginosa biofilm production inhibition. This newly engineered bacteriophage induced a significant decrease in bacterial count as well as an important inhibition of quorumsensing activity. ${ }^{49}$

\section{Proof of concept in animal models of pneumonia}

The first use of animal experiments to assess the efficacy of bacteriophage-based therapy was related by d'Herelle against Pasteurella multocida. In 1919, while epidemic fowl cholera was responsible for poultry mortality in France, he gave water that contained phages active against the bacterium responsible for the disease to the non-diseased animals and noticed that phage-pretreated animals did not contract the infection. In the same way, he obtained efficacy against hemorrhagic septicemia in cattle. D'Herelle pursued animal studies to assess the interaction of phages with the mammalian immune system. He observed that guinea pig leukocytes incubated in the presence of Shigella bacteria and bacteriophages had enhanced phagocytic power compared to incubation with the bacteria alone. Later, and until recently, ${ }^{50}$ studies in guinea pigs and mice produced discordant data, suggesting that the effect of phages on mammalian phagocytic activity against bacteria may depend on numerous factors, including the concentration of the phages, the bacterial species, the duration of the experiment, and the type of phagocytic cell studied.

Animal studies are of special interest to assess bacteriophage toxicity and diffusion in mammalian organisms. Routes and delay of bacteriophage penetration in vertebrates have been extensively evaluated in various animal species and are reviewed in detail by Dabrowska et al. ${ }^{51}$ Briefly, whatever 
the route of inoculation, bacteriophages exhibit a rapid bloodstream spread and multiorgan diffusion ability, including into the lung and the brain. After any oral, nasal, aerosol, intra-uterine, skin, or other route of administration, the delay before detection of phages in the blood is short (15 minutes to 1 hour), except after intranasal administration, which has been reported to take approximately 24 hours to diffuse into systemic circulation. No toxicity, organ dysfunction, or lesion was observed with these different routes of administration. After a single dose, bacteriophage clearance takes several days. As one could expect, the persistence of bacteriophages in an animal's body is enhanced when concomitant infection with sensitive bacteria exists.

The subject of respiratory tract infections started to be questioned in the 2000s. Huff and colleagues conducted a series of experiments to treat $E$. coli respiratory infections in broiler chickens. ${ }^{52}$ They demonstrated that an aerosol spray given just after bacterial inoculation with $10^{4}$ colony-forming units (CFU) was able to reduce the mortality from $50 \%$ to $20 \%$. Approximately 10 years later, a few animal studies were specifically designed to assess clinically relevant endpoints in pneumonia with the benefits of recent experimental techniques including in vivo light imaging and fluorescent confocal microscopy. However, experimental data from in vivo animal studies are still scarce in the field of pneumonia, and those that do exist are from studies mainly conducted in mice with $P$. aeruginosa,${ }^{53-56}$ Klebsiella pneumoniae, ${ }^{57}$ or Burkholderia cenocepacia ${ }^{58}$ As far as we are aware, only two studies focused on CF and P. aeruginosa ${ }^{54,55}$ (Table 2).

Whatever the animal species or the bacteriophage, all reports converge to state that the efficacy of bacteriophagebased therapy in lung infection is time and dose dependent. Indeed, nearly all studies assessing this endpoint show that efficacy relies on the time frame of the treatment (within the first 2 hours postinfection), and preventive administration should be considered ( 24 hours before bacterial challenge). ${ }^{53}$ In a lethal mouse model of pneumonia using an MDR $P$. aeruginosa strain from CF patients, Morello et a ${ }^{54}$ reported that a single intranasal administration of the bacteriophages PAK-P or P3-CHA resulted in survival rates of $90 \%-100 \%$ and reduced pathological damage when given within 2 hours postinfection. In addition, a preventive effect was obtained with the bacteriophage P3-CHA when given 4 days prior to infection, showing $100 \%$ protection at a dose of $3 \times 10^{8}$ plaque-forming units.

The bacteriophage-to-bacterium ratio appears to influence treatment efficacy. For example, in the preliminary part of a study published by Debarbieux et $\mathrm{a}^{53}$ all mice

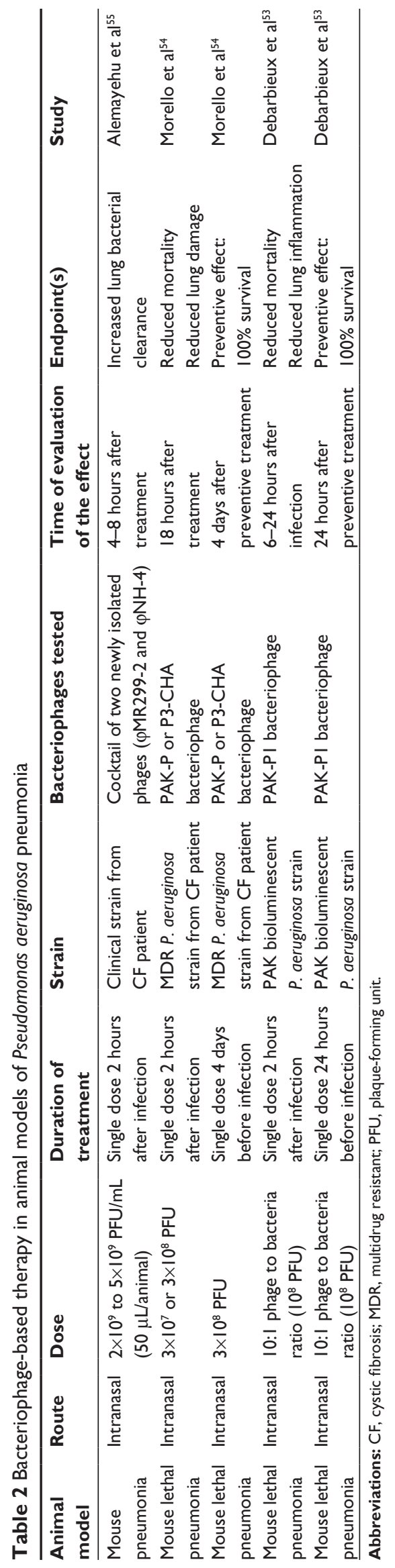


treated intranasally with a phage-to-bacterium ratio of 1:10 died within 5 days following infection with $10^{7} \mathrm{CFU}$ $P$. aeruginosa, whereas mice treated with ratios of $1: 1$ and 10:1 survived until 12 days. Similarly, other studies using $K$. pneumoniae or B. cenocepacia showed the efficacy of bacteriophage-based therapy in reducing lung bacterial burden when given early at high amounts. ${ }^{58,59}$

Very recently, efficacy with delayed treatment was reported, ${ }^{60}$ which is of major interest for its clinical relevance. In an immunocompromised (cyclophosphamide-induced leukopenia) mouse model of antibiotic-resistant Burkholderia cepacia from CF lung infection, Semler et al showed efficacy of a bacteriophage therapy (five bacteriophage strains including Myoviridae and Podoviridae) delivered via aerosol 24 hours postinfection. ${ }^{60}$ Two days after treatment ( 3 days postinfection), bacterial lung culture decreased by 2.5 to $4.0 \mathrm{log}$, the aerosol route showing better results than the intraperitoneal route. Liposomes can also be used as a delivery vehicle for phages with the advantage of delayed efficiency, as recently reported in a mouse model of $K$. pneumoniae lobar pneumonia with both therapeutic and prophylactic administration. ${ }^{57}$

Whether bacteriophages are still efficient against mucoid strains is a major concern. Alemayehu et $\mathrm{al}^{55}$ assessed the effect on the biofilm of two bacteriophages active against $P$. aeruginosa, the newly isolated bacteriophage NH-4 and the bacteriophage MR299-2, both by in vitro and in vivo experiments. For their in vivo study, after isolation of several $P$. aeruginosa strains from CF patients' sputum, they lux tagged a mucoid strain and a non-mucoid strain. BALB/c mice were infected intranasally (approximately $10^{7} \mathrm{CFU} /$ mouse), and after 2 hours, animals were inoculated with a phage-mix suspension at approximately $10^{8}$ plaque-forming units per animal. Bioluminescent imaging performed from 2 to 6 hours after infection (maximal level at 6 hours) showed a significant reduction in fluorescence in the phage-treated mice, regardless of whether they were infected with a mucoid or non-mucoid strain. These data offer interesting perspectives for future treatment of CF patients who often chronically harbor mucoid strains.

\section{Summary}

Numerous animal studies argue for a preventive or curative treatment of $P$. aeruginosa lung infection with bacteriophages, even when mucoid strains and biofilm formation are concerned. However, the preferred route of administration, the dose, the duration of treatment, co-treatment with antibiotics, and the choice of a single agent or of a host-adapted or preexisting cocktail are still unclear. In the absence of standardization, comparison between studies is sometimes difficult. In addition, because studies in small animals have a short time of observation compared to chronic lung infections in humans, the question of whether bacteriophage-based therapy may favor transduction of virulence factors would need to be assessed in vivo. CF patients often have polymicrobial lung colonization and even anaerobic flora perhaps hidden by $P$. aeruginosa. ${ }^{61}$ To our knowledge, no animal study has yet assessed the value of bacteriophage-based therapy in targeting multiple bacterial species.

Importantly, the clearance and diffusion of therapeutic agents deep in the respiratory airways of CF patients need to be considered. The effectiveness of bacteriophages to treat infected CF-like mouse lungs would be interesting to assess in, for example, the recent mouse model of pulmonary deficiency developed by Zhou et al. ${ }^{62}$ Additionally, before delivery to patients, animal models would be of great interest to determine how bacteriophages interact with eukaryotic cells by, for example, using intravital imaging technology.

\section{Clinical studies and therapeutic perspectives \\ Safety and efficacy of bacteriophages}

Studies from Eastern countries have offered sufficient evidence to support the use of bacteriophages in controlled studies in Western countries. The prerequisite to these studies, and, more largely, to open the door of the therapeutic arsenal to bacteriophage-based therapy, is the affirmation of their safety. Indeed, the major concern is that bacteriophages are viruses, and it is difficult to override this image of human pathogens. However, phage safety and efficacy have been recently investigated. ${ }^{63}$ In that study, the authors designed a cocktail of lytic phages active against $P$. aeruginosa and $S$. aureus clinical isolates from burn patients with infected wounds. Prior to its clinical use, the cocktail was tested for multiple parameters including stability, pyrogenicity, sterility, cytotoxicity, antibacterial efficacy with specific activity against the targeted bacteria, lytic nature, the absence of temperate bacteriophages, and the absence of toxin-coding genes. This first step was recently supported in the Evaluation of Phage Therapy for the Treatment of Escherichia Coli and Pseudomonas Aeruginosa Wound Infections in Burned Patients - "Phagoburn" - study by the European Ethics Committees, and its funding was provided by the European Commission. Other controlled studies using bacteriophages in the treatment of infected wounds ${ }^{14}$ and chronic otitis ${ }^{16}$ 
demonstrated encouraging results and showed the safety of the bacteriophages.

\section{Clinical data in CF patients}

The experimental treatment of pneumonia or airway bacterial colonization in $\mathrm{CF}$ patients is until today limited to case reports. ${ }^{64}$ For example, in a 5-year-old child with failure of multiple antibiotic therapies and chronic airway colonization by $P$. aeruginosa, aerosolized bacteriophages from the Pyophage cocktail (from the Georgian Eliava institute) targeting $P$. aeruginosa were used three times a day, 6 to 10 days per month during 3 consecutive months. The authors describe an improvement of the clinical status with facilitation of expectoration and weight gain. After the third round of aerosols, $P$. aeruginosa was no longer identified in the patient's sputum. More recently, between 2007 and 2010, a study was performed on eight CF patients in Tbilisi. Patients received bacteriophages as aerosols for 6 to 10 days along with conventional antibiotic treatment, mucus thinners, and vitamins. The authors reported a decrease in the concentration of bacteria in the sputum of patients and a decrease in the need for antibiotic treatments. ${ }^{65}$ These studies are encouraging, and it seems that bacteriophages are able to reduce $P$. aeruginosa infections. However, due to their lack of standardized design and the few number of patients included, it is difficult to draw conclusions on the effectiveness of bacteriophages for this indication. Larger clinical trials, especially with randomized designs, are necessary to determine the efficacy of bacteriophage-based therapy in $P$. aeruginosa pneumonia in CF patients.

One of the main elements underlined by these studies is the therapeutic potential of bacteriophage-based therapy in CF patients using a local application by the nebulized route. Indeed, this route of administration of antibiotics is commonly used in CF patients because of the high local concentration of the agents at the infection site and the supposed low systemic diffusion. In this context, dry-powder systems for inhalation have been developed and tested ${ }^{66}$ with a phage cocktail targeting the $B$. cepacia complex and $P$. aeruginosa. This technique offers the possibility of obtaining a lyophilized solution containing phages with controlled titers and lytic activity. Using a mouth-throat replica, the nebulization technique allowed a significant inhaled fraction of bacteriophages to be distributed throughout the experimental system. Nebulization of a lyophilized solution of bacteriophages could be a potentially attractive alternative to the traditional inhaled chemotherapeutic treatments of patients with CF colonized by $P$. aeruginosa.

\section{Limits}

Although the clinical data and technical perspectives regarding bacteriophage treatment in $\mathrm{CF}$ pneumonia seem particularly interesting, the scarcity of the literature concerning specifically CF strains and patients limits for now the conclusions that can be drawn for phage therapy for this indication. Indeed, few clinical data are available. In the past, one of the main limits to the development of bacteriophage use in Western countries was the report of therapeutic failures. Thereby, the antibacterial efficacy of bacteriophages in CF patients can only be supposed from clinical case reports and from animal and in vitro studies. The only well-designed clinical studies in existence focus on wound infections, so extrapolation to the respiratory tract seems hazardous. On the other hand, bacteriophages, especially in their lysogenic lifestyle, contribute significantly to bacterial genome alterations and could be vehicles of the resistome in $\mathrm{CF} .{ }^{67}$ In addition, the question of whether bacteria develop resistance against phages requires more profound investigations.

\section{Conclusion and perspectives}

At the time of a scarcity of new antibiotics against MDR infections, bacteriophages are potential new anti-infectious treatments that may be of particular interest against bacterial flora from $\mathrm{CF}$ patients given the frequency of MDR bacteria in this population. Today, evidence is growing to support bacteriophage-based therapy in such patients, especially in $P$. aeruginosa infections. Phage cocktails targeting $P$. aeruginosa are already available and new phages can easily be identified in the environment. Phage specificity against clinical strains from CF sputum has been proven, as well as phages' possible synergy with antibiotics. Phage activity against biofilms, which are particularly involved in CF persistent infections, is also of particular interest. The results of animal studies seem to agree with in vitro findings. The inhaled route of administration has been demonstrated to be suitable for bacteriophage aerosol administration. With the development of molecular biology, bacterial genome sequencing may allow the identification of putative target genes associated with bacteriophage activity. With the beginning of the first randomized controlled study using bacteriophage treatments in infected wounds and the demonstration of their safety in clinical use, the road seems open for the first clinical trials evaluating the efficacy of bacteriophages in the treatment of $P$. aeruginosa infections in CF patients. However, many features remain to be clarified, such as the route of administration, the timing of treatment in the evolution of chronic 
infections, the standardization of delivery (dose, frequency), administration with antibiotics, and the risk for the emergence of lysogenic forms with horizontal gene-transfer abilities. Even if the main limitation is that no well-designed clinical study has been conducted, physicians and researchers cannot ignore bacteriophages in the search for innovative treatments for $P$. aeruginosa in CF patients.

\section{Disclosure}

The authors declare no conflicts of interest in this work.

\section{References}

1. Ciofu O, Tolker-Nielsen T, Jensen PO, Wang H, Høiby N. Antimicrobial resistance, respiratory tract infections and role of biofilms in lung infections in cystic fibrosis patients. Adv Drug Deliv Rev. 2014. Epub December 2.

2. Emerson J, Rosenfeld M, McNamara S, Ramsey B, Gibson RL. Pseudomonas aeruginosa and other predictors of mortality and morbidity in young children with cystic fibrosis. Pediatr Pulmonol. 2002; 34(2):91-100.

3. Li Z, Kosorok MR, Farrell PM, et al. Longitudinal development of mucoid Pseudomonas aeruginosa infection and lung disease progression in children with cystic fibrosis. JAMA. 2005;293(5):581-588.

4. Langton Hewer SC, Smyth AR. Antibiotic strategies for eradicating Pseudomonas aeruginosa in people with cystic fibrosis. Cochrane Database Syst Rev. 2009;(4)CD004197.

5. Hansen CR, Pressler T, Høiby N. Early aggressive eradication therapy for intermittent Pseudomonas aeruginosa airway colonization in cystic fibrosis patients: 15 years experience. J Cyst Fibros. 2008;7(6): 523-530.

6. Ciofi Degli AM, Bernaschi P, Carletti M, et al. An outbreak of extremely drug-resistant Pseudomonas aeruginosa in a tertiary care pediatric hospital in Italy. BMC Infect Dis. 2014;14:494.

7. Bassetti M, Ginocchio F, Mikulska M. New treatment options against gram-negative organisms. Crit Care. 2011;15(2):215.

8. Hraiech S, Brégeon F, Brunel JM, et al. Antibacterial efficacy of inhaled squalamine in a rat model of chronic Pseudomonas aeruginosa pneumonia. J Antimicrob Chemother. 2012;67(10):2452-2458.

9. Hraiech S, Hiblot J, Lafleur J, et al. Inhaled lactonase reduces Pseudomonas aeruginosa quorum sensing and mortality in rat pneumonia. PLoS One. 2014;9(10):e107125.

10. Hurley MN, Cámara M, Smyth AR. Novel approaches to the treatment of Pseudomonas aeruginosa infections in cystic fibrosis. Eur Respir J. 2012;40(4):1014-1023.

11. Harper DR, Enright MC. Bacteriophages for the treatment of Pseudomonas aeruginosa infections. J Appl Microbiol. 2011;111(1):1-7.

12. Saussereau E, Vachier I, Chiron R, et al. Effectiveness of bacteriophages in the sputum of cystic fibrosis patients. Clin Microbiol Infect. 2014;20(12):O983-O990.

13. Chanishvili N. Phage therapy - history from Twort and d'Herelle through Soviet experience to current approaches. Adv Virus Res. 2012;83:3-40.

14. Rose T, Verbeken G, Vos DD, et al. Experimental phage therapy of burn wound infection: difficult first steps. Int J Burns Trauma. 2014;4(2): 66-73.

15. Rhoads DD, Wolcott RD, Kuskowski MA, Wolcott BM, Ward LS, Sulakvelidze A. Bacteriophage therapy of venous leg ulcers in humans: results of a phase I safety trial. J Wound Care. 2009;18(6):237-238, 240-243.

16. Wright A, Hawkins CH, Anggård EE, Harper DR. A controlled clinical trial of a therapeutic bacteriophage preparation in chronic otitis due to antibiotic-resistant Pseudomonas aeruginosa; a preliminary report of efficacy. Clin Otolaryngol. 2009;34(4):349-357.
17. Reardon S. Phage therapy gets revitalized. Nature. 2014;510(7503): $15-16$.

18. Berthelot P, Grattard F, Mallaval FO, Ros A, Lucht F, Pozzetto B. [Epidemiology of nosocomial infections due to Pseudomonas aeruginosa, Burkholderia cepacia and Stenotrophomonas maltophilia.] Pathol Biol (Paris). 2005;53(6):341-348. French.

19. Drenkard E, Ausubel FM. Pseudomonas biofilm formation and antibiotic resistance are linked to phenotypic variation. Nature. 2002;416(6882):740-743.

20. Costerton JW, Stewart PS, Greenberg EP. Bacterial biofilms: a common cause of persistent infections. Science. 1999;284(5418): 1318-1322.

21. Hoyle BD, Alcantara J, Costerton JW. Pseudomonas aeruginosa biofilm as a diffusion barrier to piperacillin. Antimicrob Agents Chemother. 1992;36(9):2054-2056.

22. Lewis K. Persister cells, dormancy and infectious disease. Nat Rev Microbiol. 2007;5(1):48-56.

23. Gupta K, Marques CN, Petrova OE, Sauer K. Antimicrobial tolerance of Pseudomonas aeruginosa biofilms is activated during an early developmental stage and requires the two-component hybrid SagS. J Bacteriol. 2013;195(21):4975-4987.

24. Doehn JM, Fischer K, Reppe K, et al. Delivery of the endolysin Cpl-1 by inhalation rescues mice with fatal pneumococcal pneumonia. J Antimicrob Chemother. 2013;68(9):2111-2117.

25. Hanlon GW. Bacteriophages: an appraisal of their role in the treatment of bacterial infections. Int J Antimicrob Agents. 2007;30(2): $118-128$.

26. Kutter E, De Vos D, Gvasalia G, et al. Phage therapy in clinical practice: treatment of human infections. Curr Pharm Biotechnol. 2010;11(1): 69-86.

27. Ojeniyi B, Birch-Andersen A, Mansa B, Rosdahl VT, Høiby N. Morphology of Pseudomonas aeruginosa phages from the sputum of cystic fibrosis patients and from the phage typing set. An electron microscopy study. APMIS. 1991;99(10):925-930.

28. Essoh C, Blouin Y, Loukou G, et al. The susceptibility of Pseudomonas aeruginosa strains from cystic fibrosis patients to bacteriophages. PLoS One. 2013;8(4):e60575.

29. Ceyssens PJ, Lavigne R. Bacteriophages of Pseudomonas. Future Microbiol. 2010;5(7):1041-1055.

30. Selezska K, Kazmierczak M, Müsken M, et al. Pseudomonas aeruginosa population structure revisited under environmental focus: impact of water quality and phage pressure. Environ Microbiol. 2012;14(8): 1952-1967.

31. Friman VP, Ghoul M, Molin S, Johansen HK, Buckling A. Pseudomonas aeruginosa adaptation to lungs of cystic fibrosis patients leads to lowered resistance to phage and protist enemies. PLoS One. 2013;8(9): e75380.

32. Hraiech S, Roch A, Lepidi H, et al. Impaired virulence and fitness of a colistin-resistant clinical isolate of Acinetobacter baumannii in a rat model of pneumonia. Antimicrob Agents Chemother. 2013;57(10):5120-5121.

33. Koskella B, Lin DM, Buckling A, Thompson JN. The costs of evolving resistance in heterogeneous parasite environments. Proc Biol Sci. 2012; 279(1735):1896-1903.

34. James CE, Fothergill JL, Kalwij H, et al. Differential infection properties of three inducible prophages from an epidemic strain of Pseudomonas aeruginosa. BMC Microbiol. 2012;12:216.

35. Hayashi T, Baba T, Matsumoto H, Terawaki Y. Phage-conversion of cytotoxin production in Pseudomonas aeruginosa. Mol Microbiol. 1990; 4(10):1703-1709.

36. Winstanley C, Langille MG, Fothergill JL, et al. Newly introduced genomic prophage islands are critical determinants of in vivo competitiveness in the Liverpool Epidemic Strain of Pseudomonas aeruginosa. Genome Res. 2009;19(1):12-23.

37. Fu W, Forster T, Mayer O, Curtin JJ, Lehman SM, Donlan RM. Bacteriophage cocktail for the prevention of biofilm formation by Pseudomonas aeruginosa on catheters in an in vitro model system. Antimicrob Agents Chemother. 2010;54(1):397-404. 
38. Hanlon GW, Denyer SP, Olliff CJ, Ibrahim LJ. Reduction in exopolysaccharide viscosity as an aid to bacteriophage penetration through Pseudomonas aeruginosa biofilms. Appl Environ Microbiol. 2001; 67(6):2746-2753.

39. Costerton JW, Cheng KJ, Geesey GG, et al. Bacterial biofilms in nature and disease. Annu Rev Microbiol. 1987;41:435-464.

40. Donlan RM. Biofilms and device-associated infections. Emerg Infect Dis. 2001;7(2):277-281

41. Singh PK, Schaefer AL, Parsek MR, Moninger TO, Welsh MJ, Greenberg EP. Quorum-sensing signals indicate that cystic fibrosis lungs are infected with bacterial biofilms. Nature. 2000; 407(6805):762-764.

42. Verma V, Harjai K, Chhibber S. Structural changes induced by a lytic bacteriophage make ciprofloxacin effective against older biofilm of Klebsiella pneumoniae. Biofouling. 2010;26(6):729-737.

43. Glonti T, Chanishvili N, Taylor PW. Bacteriophage-derived enzyme that depolymerizes the alginic acid capsule associated with cystic fibrosis isolates of Pseudomonas aeruginosa. J Appl Microbiol. 2010;108(2): 695-702.

44. Sulakvelidze A, Alavidze Z, Morris JG Jr. Bacteriophage therapy. Antimicrob Agents Chemother. 2001;45(3):649-659.

45. Lu TK, Collins JJ. Engineered bacteriophage targeting gene networks as adjuvants for antibiotic therapy. Proc Natl Acad Sci U S A. 2009; 106(12):4629-4634.

46. Escobar-Páramo P, Gougat-Barbera C, Hochberg ME. Evolutionary dynamics of separate and combined exposure of Pseudomonas fluorescens SBW25 to antibiotics and bacteriophage. Evol Appl. 2012; 5(6):583-592.

47. Torres-Barceló C, Arias-Sánchez FI, Vasse M, Ramsayer J, Kaltz O, Hochberg ME. A window of opportunity to control the bacterial pathogen Pseudomonas aeruginosa combining antibiotics and phages. PLoS One. 2014;9(9):e106628.

48. Coulter LB, McLean RJ, Rohde RE, Aron GM. Effect of bacteriophage infection in combination with tobramycin on the emergence of resistance in Escherichia coli and Pseudomonas aeruginosa biofilms. Viruses. 2014;6(10):3778-3786.

49. Pei R, Lamas-Samanamud GR. Inhibition of biofilm formation by T7 bacteriophages producing quorum-quenching enzymes. Appl Environ Microbiol. 2014;80(17):5340-5348.

50. Górski A, Międzybrodzki R, Borysowski J, et al. Phage as a modulator of immune responses: practical implications for phage therapy. $A d v$ Virus Res. 2012;83:41-71.

51. Dabrowska K, Switała-Jelen K, Opolski A, Weber-Dabrowska B, Gorski A. Bacteriophage penetration in vertebrates. J Appl Microbiol. 2005;98(1):7-13.

52. Huff WE, Huff GR, Rath NC, Balog JM, Donoghue AM. Evaluation of aerosol spray and intramuscular injection of bacteriophage to treat an Escherichia coli respiratory infection. Poult Sci. 2003;82(7): 1108-1112.

53. Debarbieux L, Leduc D, Maura D, et al. Bacteriophages can treat and prevent Pseudomonas aeruginosa lung infections. J Infect Dis. 2010; 201(7):1096-1104.
54. Morello E, Saussereau E, Maura D, Huerre M, Touqui L, Debarbieux L. Pulmonary bacteriophage therapy on Pseudomonas aeruginosa cystic fibrosis strains: first steps towards treatment and prevention. PLoS One. 2011;6(2):e16963

55. Alemayehu D, Casey PG, McAuliffe O, et al. Bacteriophages $\phi M R 299-2$ and $\phi \mathrm{NH}-4$ can eliminate Pseudomonas aeruginosa in the murine lung and on cystic fibrosis lung airway cells. MBio. 2012;3(2):e00029-12.

56. Henry M, Lavigne R, Debarbieux L. Predicting in vivo efficacy of therapeutic bacteriophages used to treat pulmonary infections. Antimicrob Agents Chemother. 2013;57(12):5961-5968.

57. Singla S, Harjai K, Katare OP, Chhibber S. Bacteriophage-Loaded Nanostructured Lipid Carrier: Improved Pharmacokinetics Mediates Effective Resolution of Klebsiella pneumoniae-Induced Lobar Pneumonia. J Infect Dis. 2015. Epub January 20.

58. Carmody LA, Gill JJ, Summer EJ, et al. Efficacy of bacteriophage therapy in a model of Burkholderia cenocepacia pulmonary infection. J Infect Dis. 2010;201(2):264-271.

59. Chhibber S, Kaur S, Kumari S. Therapeutic potential of bacteriophage in treating Klebsiella pneumoniae B5055-mediated lobar pneumonia in mice. J Med Microbiol. 2008;57(Pt 12):1508-1513.

60. Semler DD, Goudie AD, Finlay WH, Dennis JJ. Aerosol phage therapy efficacy in Burkholderia cepacia complex respiratory infections. Antimicrob Agents Chemother. 2014;58(7):4005-4013.

61. Bittar F, Rolain JM. Detection and accurate identification of new or emerging bacteria in cystic fibrosis patients. Clin Microbiol Infect. 2010;16(7):809-820.

62. Zhou Z, Duerr J, Johannesson B, et al. The ENaC-overexpressing mouse as a model of cystic fibrosis lung disease. J Cyst Fibros. 2011;10 Suppl 2: S172-S182.

63. Merabishvili M, Pirnay JP, Verbeken G, et al. Quality-controlled smallscale production of a well-defined bacteriophage cocktail for use in human clinical trials. PLoS One. 2009;4(3):e4944.

64. Kutateladze M, Adamia R. Phage therapy experience at the Eliava Institute. Med Mal Infect. 2008;38(8):426-430.

65. Kutateladze M, Adamia R. Bacteriophages as potential new therapeutics to replace or supplement antibiotics. Trends Biotechnol. 2010 28(12):591-595.

66. Golshahi L, Lynch KH, Dennis JJ, Finlay WH. In vitro lung delivery of bacteriophages KS4-M and $\Phi \mathrm{KZ}$ using dry powder inhalers for treatment of Burkholderia cepacia complex and Pseudomonas aeruginosa infections in cystic fibrosis. J Appl Microbiol. 2011;110(1):106-117.

67. Rolain JM, Fancello L, Desnues C, Raoult D. Bacteriophages as vehicles of the resistome in cystic fibrosis. J Antimicrob Chemother. 2011; 66(11):2444-2447.

68. Northrop JH. Growth and phage production of B. megatherium. I. Growth of cells after infection with C phage. II. Rate of growth, phage yield, and RNA content of cells. III. Effect of various substances on growth rate and phage production. J Gen Physiol. 1953;36(4):581-599.
Drug Design, Development and Therapy

\section{Publish your work in this journal}

Drug Design, Development and Therapy is an international, peerreviewed open-access journal that spans the spectrum of drug design and development through to clinical applications. Clinical outcomes, patient safety, and programs for the development and effective, safe, and sustained use of medicines are a feature of the journal, which

\section{Dovepress}

has also been accepted for indexing on PubMed Central. The manuscript management system is completely online and includes a very quick and fair peer-review system, which is all easy to use. Visit http://www.dovepress.com/testimonials.php to read real quotes from published authors. 